Article

\title{
Visual-Based Multi-Section Welding Path Generation Algorithm
}

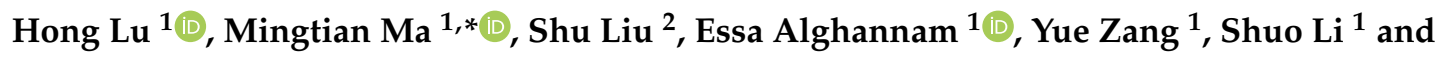 \\ Weixin Zhang ${ }^{1}$ \\ 1 School of Mechanical and Electronic Engineering, Wuhan University of Technology, Wuhan 430070, China; \\ landzh@whut.edu.cn (H.L.); essaalghannam@whut.edu.cn (E.A.); zangyue@whut.edu.cn (Y.Z.); \\ lishuo0708@163.com (S.L.); whut_zwx@163.com (W.Z.) \\ 2 China Construction Science and Industry Corporation Limited, Wuhan 430070, China; liushu@cscec.com \\ * Correspondence: wxmmt100@163.com
}

Received: 18 June 2020; Accepted: 8 July 2020; Published: 11 July 2020

\begin{abstract}
As an important form of additive manufacturing, welding is widely used in steel components welding work of construction, shipbuilding and other fields. In this study, an intelligent welding path generation algorithm based on multi-section interpolation is proposed in order to deal with non-standard multi-pass welding grooves which are difficult to be handled by automatic welding equipment in the construction site. Firstly, the non-standard grooves are classified and the reasons for their occurrence are discussed. Secondly, an automatic welding additive manufacturing system framework is discussed and an appropriate detection method is selected. Then, combining with the welding standard of non-standard grooves and the characteristics of the welding process, a multi-section interpolation-based welding path generation algorithm is proposed. Finally, a visual experiment platform was built to detection the typical non-standard groove and the welding experiment is implemented to verify the feasibility of the algorithm. According to the path generated by the algorithm, the welded steel components test plate meets the actual engineering standard after quality inspection. The experimental results and simulation results conclude the algorithm can be used to generate the welding path of the non-standard groove.
\end{abstract}

Keywords: welding; vision system; non-standard groove; path generation algorithm

\section{Introduction}

Welding is a manufacturing process and technology for joining alloys or other metal materials by means of heating, high temperature or high pressure. Gas shielded welding has become one of the most important welding methods for ferrous metal [1-3]. It is widely used in civil engineering, shipbuilding, and heavy industry because of its low cost, good weld formation and high-quality welded joints without internal defects. In the construction and manufacturing industry, a large number of steel components are put into use, which need welding to assemble and fix. The ditch with a certain geometry shape processed and assembled at the position to be welded is the welding groove. The quality of groove welding determines the reliability and safety of steel component assembly. Generally, the cross-section shape and geometric size of groove have been preset in the design and processing of steel components. However, due to the deformation of steel components, assembly error and other factors, the cross-section shape and geometric size of groove will change unpredictably in actual assembly, forming a non-standard groove. Although the application of automatic welding equipment in workshop-level processing based on a process database and teaching system has become mature [4], it is still unable to adapt to the irregular and unpredictable non-standard groove welding operation with irregular shape and size changes. The wrong welding path will lead to porosity, 
undercut, incomplete welding and other problems, which will affect the welding quality. The welding work of a non-standard groove still relies on a welding operators' experience. A system that can detect the shape and size changes of a non-standard groove and the research of multi-pass trajectory planning based on groove geometric parameters are of great significance for automatic welding.

Many scholars have studied the detection of welding grooves. Kevin Micallef proposed a low-cost vision sensor for locating complex shape welds [5]. Xu realized real-time seam tracking control during welding robot process based on passive vision sensor [6]. A welding path measuring system is developed by Drago Bračun in order to achieve better understanding of the welding execution of the critical welds or weld deposits in multi-pass and repair welding [7]. The detection and tracking technology of a single-pass weld is not suitable for large grooves with multi-pass welding [8]. A conventional visual inspection method cannot fully extract the irregular shape and size of non-standard groove. It is necessary to determine a detection method that can fully extract the shape and size of non-standard groove to guide the subsequent path planning.

There are also many progresses in the research of multi-path trajectory planning. M. Masaharu adopted an equal area method to arrange the multi-pass welding [9]. An offline simulation system of a multi-pass basis on three-dimensional graphics is developed by Tang [10]. The existing methods for multi-pass path planning, such as that of Zhang [11], mainly aim at the situation of whole groove shape and size are consistent or those are consistent with the preset. Due to processing error, deformation and assembly error, the shape and size of the groove may change greatly compared with the preset, and the processing methods, such as tapping and heating, may also produce a more unpredictable deformation. The common path-planning algorithm is not suitable for non-standard groove, so it is necessary to design a special path-planning algorithm to guide the automatic welding equipment for welding operation [12-14].

In this paper, the non-standard groove is simply classified, and a set of visual inspection systems based on the laser triangulation principle is designed to extract the complete multi-section characteristic parameters of groove, and then the multi-pass welding path generation algorithm is imported combined with the set welding parameters, so as to obtain the movement track of the welding gun, and guide the automatic welding equipment to complete the automatic welding operation of the non-standard groove on the construction site.

\section{Problem Description}

\subsection{Introduction of Steel Component Groove}

The object of this paper is the groove of traditional steel member, which is mainly composed of carbon steel components and steel sleeve. Before welding, the relative position of steel components is determined by the connecting plate on the steel components, and the steel sleeves are fixed on the steel components by spot welding. The structure and combination mode of groove are shown in Figure 1.

The main technological characteristics of the welding process of steel components are as follows:

(1) The welding method is carbon dioxide gas-shielded welding.

(2) There are many types of grooves, including $\mathrm{K}-, \mathrm{V}-, \mathrm{U}$ - and Unilateral V-type grooves, etc.

(3) Due to machining error, assembly error, deformation and other factors, the grooves used on construction mostly have changed in geometry compared to expectations, becoming non-standard grooves. 


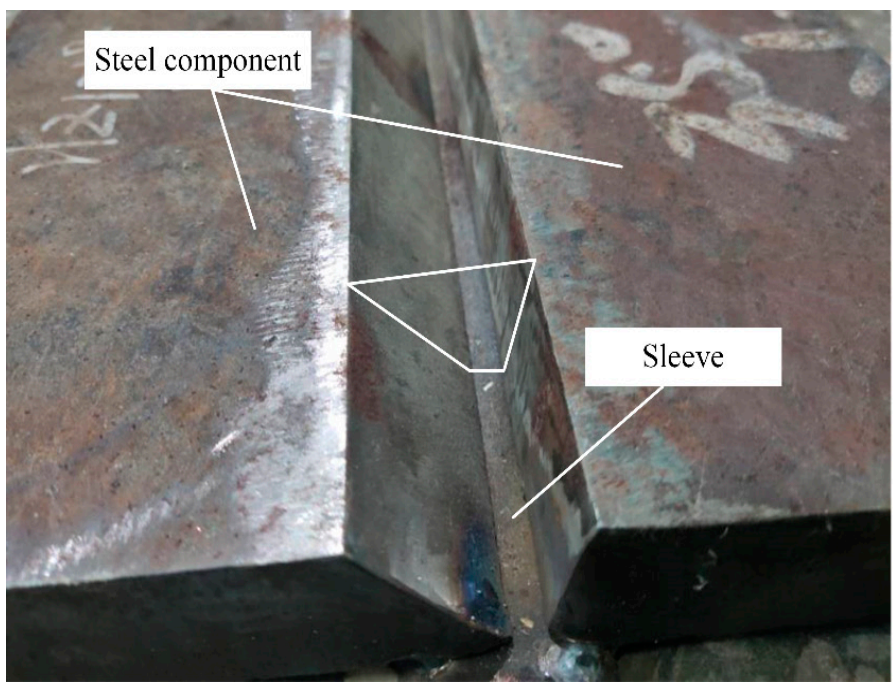

(a)

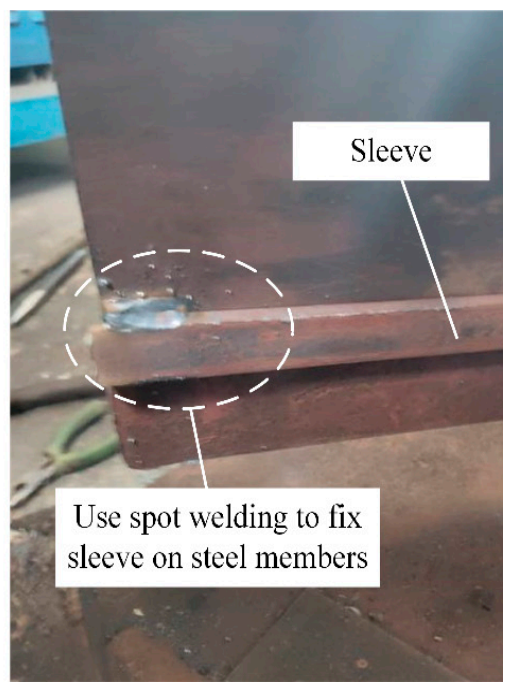

(b)

Figure 1. (a) Groove structure (The white part is the groove section shape); (b) Combination.

\subsection{Classification of Non-Standard Grooves}

The processing and assembling dimensions of steel components have independent industry standards, and grooves with standard dimensions are called standard grooves. However, due to the factors of processing and assembly, the groove shape will be distorted and the size will change in the actual construction site. Although the steel components can be straightened by heating and knocking at the site, steel components generally have large volume and weight. They will deform when they are processed, transported and hoisted. In actual assembly, they will be limited to ensure that complex steel components can be connected and fixed smoothly. The shape and size of grooves cannot be guaranteed, and most of the grooves in the construction site are non-standard grooves.

Non-standard grooves are divided into the following categories:

\section{(1) Shape distortion of groove}

The situation of shape distortion of groove is usually caused by deformation of steel member itself. The shapes of different sections along the groove direction are inconsistent. The situation of shape distortion of groove is shown in Figure 2.

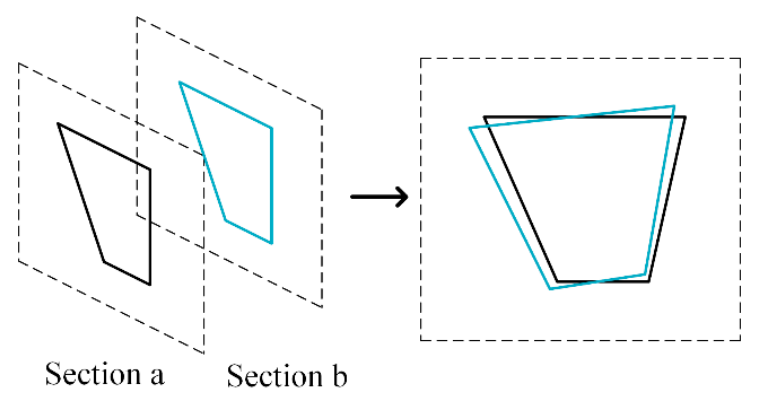

Figure 2. Non-standard grooves (shape distortion of groove).

(2) Non-uniform variation of bottom clearance changes in a small range

The situation of non-uniform variation of bottom clearance in a small range is usually caused by edge-cutting technology in machining steel components or edge collision in the transportation and lifting of steel components. In welding operation, steel components and steel sleeves need to be welded 
together. The weld path at the joint of the two is very important. It should cover steel components and steel liners at the same time. Non-uniform changes between the bottoms will increase the difficulty of operation of the automatic welding equipment. The situation of bottom clearance changes in a small range is shown in Figure 3.
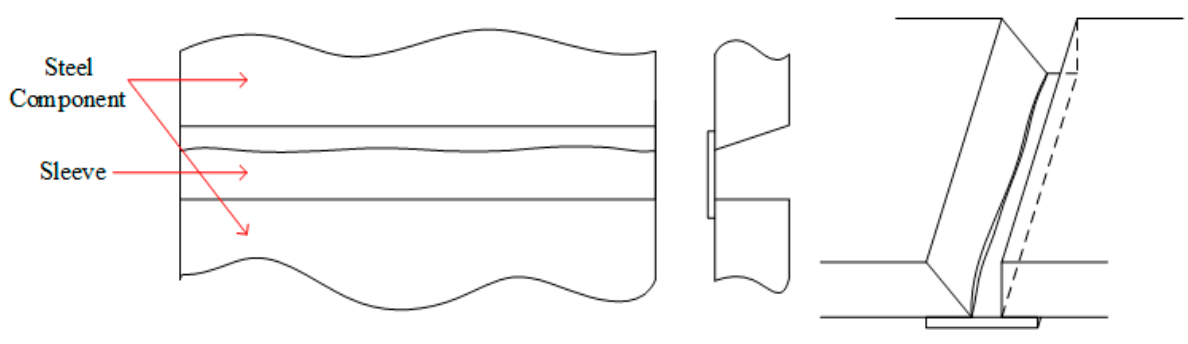

Figure 3. Non-standard grooves (non-uniform variation of bottom clearance in a small range).

(3) Unidirectional linear variation of bottom clearance

The situation of unidirectional linear variation of bottom clearance is usually caused by assemble errors of plural number steel components. In the actual assembly process, priority should be given to ensuring that steel components are fixed with each other. The inconsistent position and expectation of large steel components during assembly cause the situation of unidirectional linear variation of the bottom clearance. This is the most difficult case for automatic welding equipment to deal with. The situation of unidirectional linear variation of bottom clearance is shown in Figure 4.
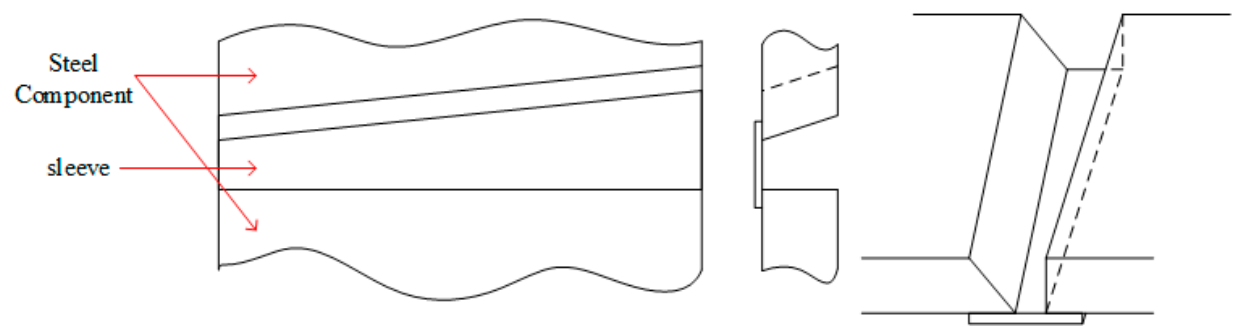

Figure 4. Non-standard grooves (unidirectional linear variation of bottom clearance).

\section{System Scheme and Detection System}

\subsection{System Scheme of Non-Standard Groove Automatic Welding and Material Additive Manufacturing System}

The situation of the unidirectional linear variation of bottom clearance is usually caused by assembly errors of plural steel components. In the actual assembly process, priority should be given to ensuring that steel components are fixed with each other. An inconsistent position and expectation of large steel components during assembly cause the situation of unidirectional linear variation of bottom clearance. This is the most difficult case for automatic welding equipment to deal with. The situation of unidirectional linear variation of bottom clearance is shown in Figure 4.

Build a non-standard groove automatic welding and material additive manufacturing system with automatic detection and welding function. The system consists of four parts: Detection, control, robot execution and welding modules. The control module carries the whole control system and handles all data operations and path generation. The robot execution module is a robot that can clamp the welding torch, receive data sent by the control module and realize the welding action. The welding module is the equipment needed for welding processing, such as welding machine, welding torch, wire feeder and protective gas. The detection module needs to be able to collect the information and characteristic parameters of the groove, and transmit the data to the control module for processing 
before the system can carry out the follow-up work. The welding additive manufacturing system framework is shown in Figure 5.

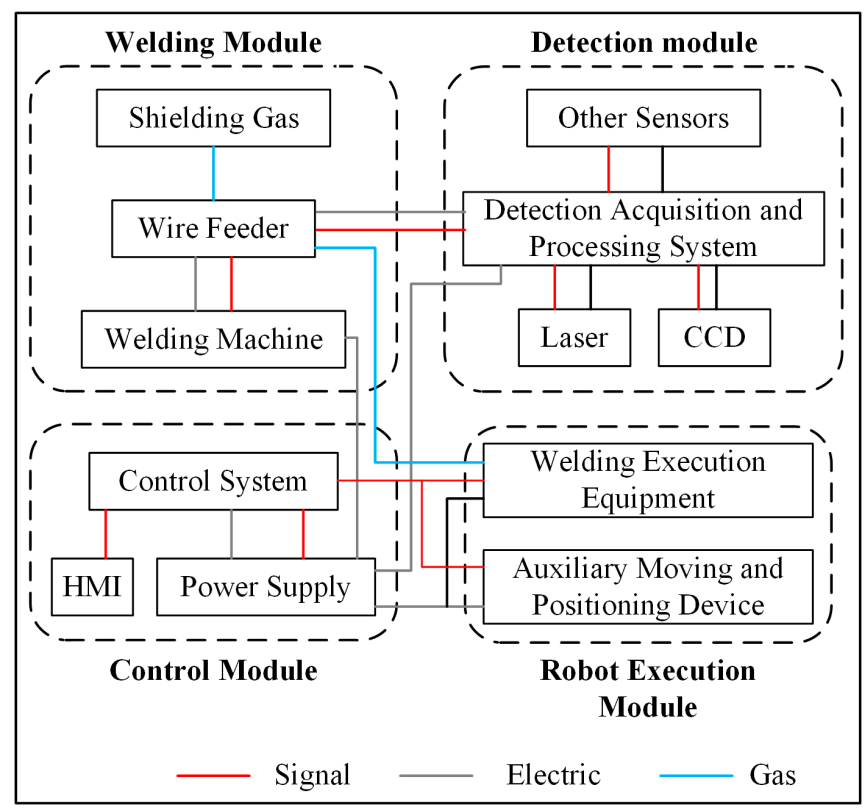

Figure 5. Welding additive manufacturing system framework.

\subsection{Detection System}

The purpose of this paper is to design a path generation algorithm which can be applied to intelligent automatic welding equipment, and to extract the feature points of multiple sections for a single groove, considering the actual use and work efficiency, finally choosing a visual method to detect the groove. The absolute coordinates extracted from the vision system carried by the robot can also be directly used for subsequent welding operations.

\subsubsection{Selection of Detection Scheme}

The detection of welded grooves of steel components has the following characteristics:

(1) The area to be monitored is a narrow rectangular area and the depth of the area does not exceed $150 \mathrm{~mm}$;

(2) The steel components themselves have been inspected for quality and their shapes are regular. There are no surface defects that will affect the welding operation;

(3) The welding operation itself will emit a lot of heat and light due to the bad illumination conditions at the work site.

The detection system is required to have the following functions:

(1) Strong ability to detect simple geometric shapes;

(2) Strong anti-jamming ability to strong light and heat;

(3) Small size, lightweight, and easy to move.

The advantages and disadvantages of various visual inspection schemes are listed in the Table 1. 
Table 1. Comparison of detection schemes.

\begin{tabular}{ccc}
\hline Detection Scheme & Advantages & Disadvantages \\
\hline Contact measurement & Simple principle & Tedious process, Low accuracy \\
Binocular vision & Efficient & Complex algorithm, Large equipment \\
Time of flight & Fast response time & Weak anti-interference ability \\
Laser triangulation & High accuracy & Strict range restriction \\
\hline
\end{tabular}

Considering the actual situation of the work site and the advantages and disadvantages of song detection method, the laser triangulation method is chosen as the final detection scheme.

\subsubsection{Principle of Laser Triangulation}

Laser triangulation is a mature method in three-dimensional detection. Mathematical triangulation is used to build models to obtain depth information.

The specific working principle is that set the laser as an active light source, projecting light onto the surface of the object to be measured, reflecting light through the imaging lens, imaging on the photosensitive chip of the industrial camera, and converting the optical signal into digital signal. According to the structure defined by the camera and the laser, the industrial camera can present the image at different angles. According to the limited freedom of the camera and laser, the height information of a single laser can be obtained by the algorithm.

The laser irradiates vertically on the surface of the object under detection. The receiver receives multiple scattering and diffuse reflected light. This irradiation method is suitable for the rough surface of the object under detection.

As shown in Figure 6, a direct triangulation diagram can be used to list the relationship between the distance on the imaging plane and the corresponding actual distance.

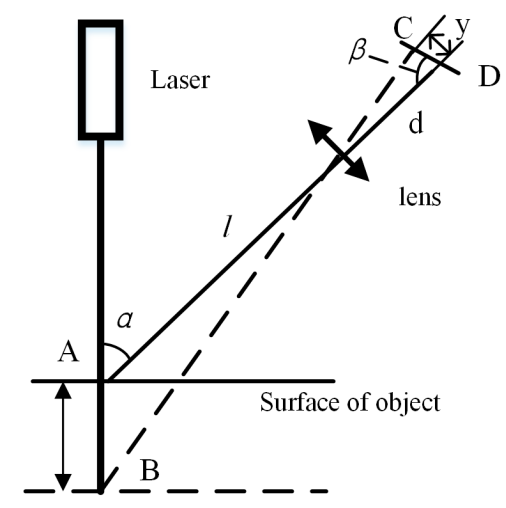

Figure 6. Principle of vertical type laser triangulation.

\subsubsection{Detection Bench and Image Process}

Fix the laser and the CCD on the bracket and prepare the calibration plate. Place the screw guide at the bottom. When detecting, the steel component test plate is placed on the movable base of the screw guide rail, and the testing begins after the calibration is completed. The method of moving the base is used to simulate the movement of the fixed detection mechanism of steel components in practical work. The detection bench is shown in Figure 7. 


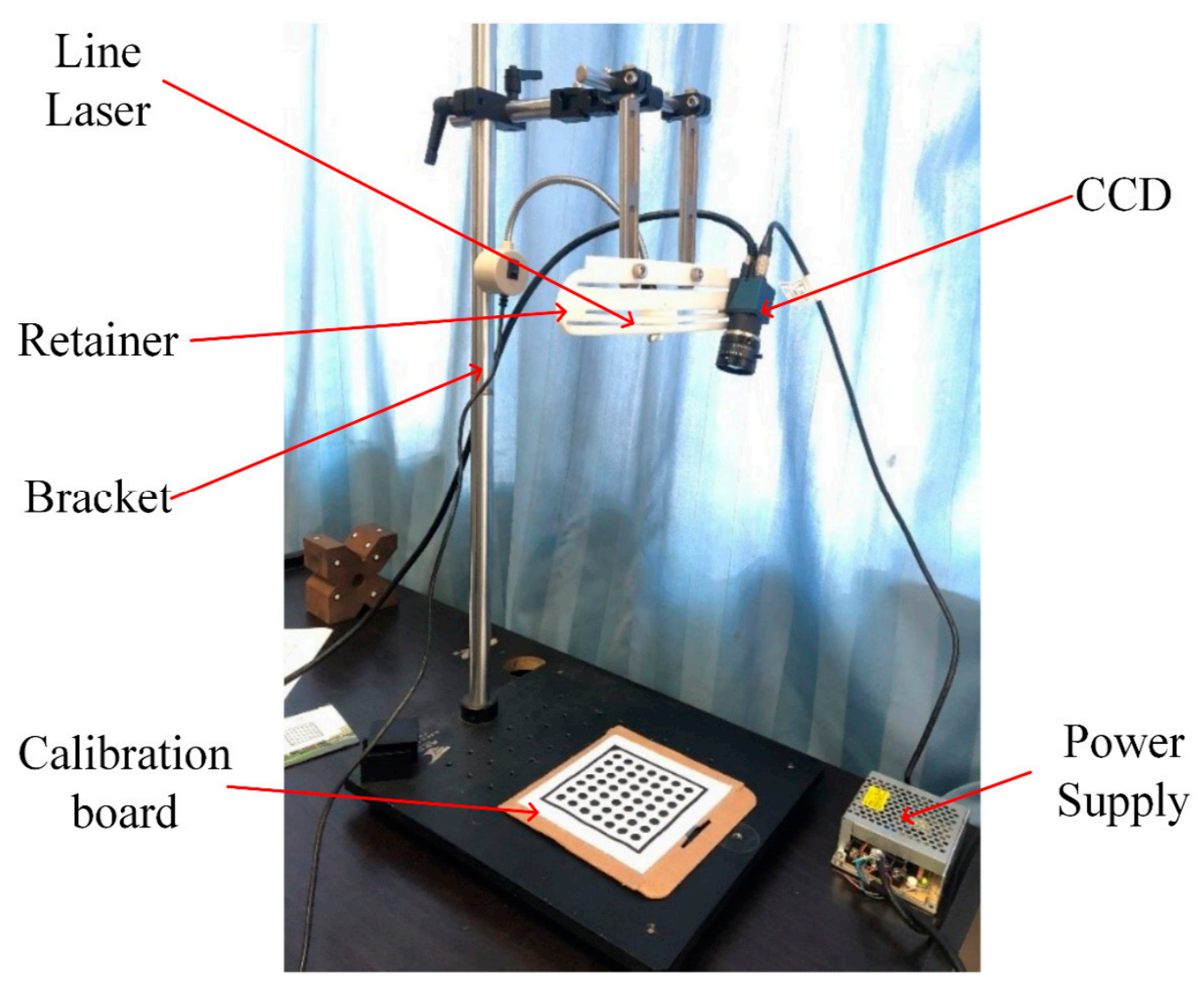

Figure 7. Detection bench for detection system.

The main steps of image processing include:

\section{(1) Clipping image}

In structured light images, structured light bars are only a small part of the image. By threshold and size segmentation, the part containing the image bars can be segmented and processed, which can greatly reduce the overall amount of computation. Specific method: The region of the strip image is converted into a horizontal minimum outer rectangle. In order to avoid the image being too compact, the clipping region is enlarged.

\section{(2) Contrast enhancement}

Linear enhancement is applied to the whole image to improve the contrast of the whole image for further processing. Based on the original method, a contrast enhancement method is proposed. The core of this method is to process the high frequency region of the image to make the generated image clearer.

(3) Noise Removal by Filtering and Threshold Segmentation

In the process of detection, noise is always everywhere. It is more obvious in the black strip image with uneven brightness. The existence of a large amount of noise increases the difficulty of strip extraction, and also affects the accuracy. Therefore, it is necessary to preprocess the image. Its purpose is to reduce image noise, combine median filtering and threshold segmentation, and carry out preliminary light strip extraction. Using median filter and threshold segmentation to remove noise. The principle of median filtering is to sort the sequence of pixels in digital image, then the gray value of the point is the median value of the gray value of the point adjacent to the pixel. 


\section{(4) Extraction of Bar Center}

After extracting the position of the strip, the center coordinates of the strip are extracted by combining the extremum method and the Hessian matrix method. The experimental results show that the algorithm can guarantee the speed of operation and has high anti-interference ability, and can realize the center location of the strip. Firstly, the extremum method finds the maximum of the gray level and locates the center of the strip initially. As the normal line of the curvature of the strip can be calculated by the Hessian matrix, the coordinates of the center point can be obtained by Taylor expansion.

(5) Extraction of Feature point coordinate

Firstly, the projection method is used to determine the approximate position of groove feature points. The projection method is to project the binary image behind the center line, count the number of white points in each line, and obtain the coordinates of the groove feature points [15-17]. Then, the least square method is used to fit the equation between you and the groove edge and the steel liner, and the obtained intersection point is the characteristic point of the groove [18-20].

\section{Generation Algorithm of Groove Welding Path Based on Multi-Section Interpolation}

The whole workflow of the technological process is shown in Figure 8.

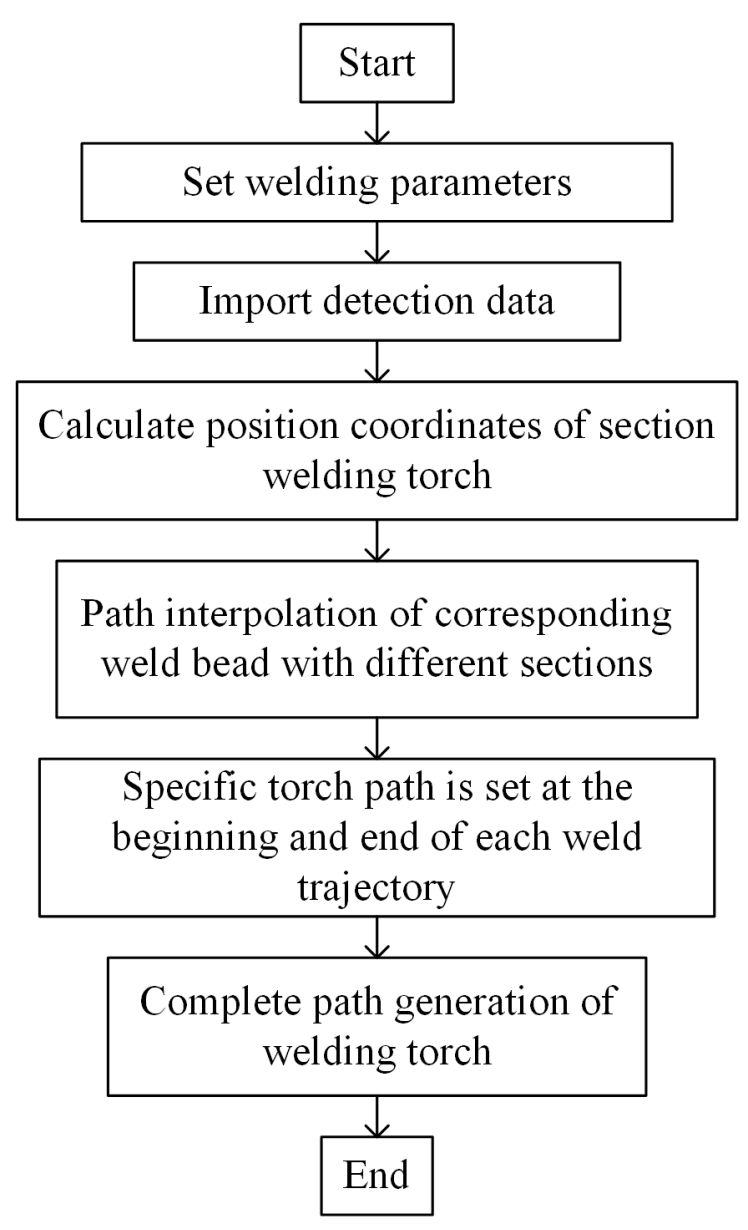

Figure 8. The workflow of technological process.

\subsection{Establishment of Groove Mathematical Model According to Welding Characteristics}

During the process of carbon dioxide arc welding work, the movements of welding torch are divided into two parts: the one part of movement $t$ is the movement flow the direction of the steel 
components for completing the whole groove's welding work, the other part of the movement is the swing perpendicular to the direction of the groove for increasing the width of the weld bead and Ensuring tight connection between steel components and steel plates.

The second part of movement is controlled by independent software, a path generation algorithm is needed to control the generation of the path of the welding torch. The decomposition of welding torch path is shown in Figure 9.

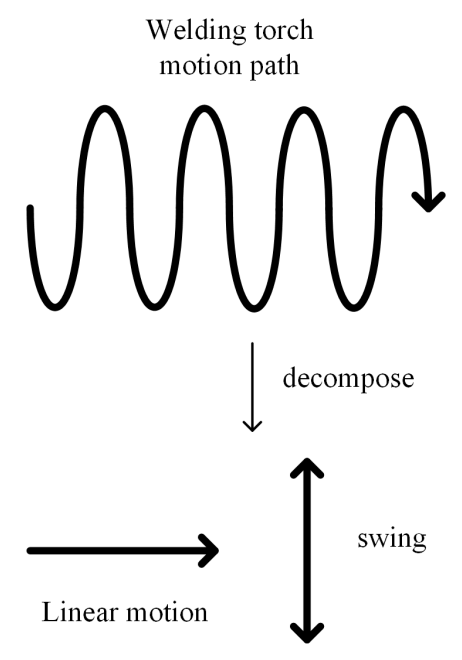

Figure 9. Decomposition of welding torch path.

The position of linear motion is judged by experience during manual operation, and the swing amplitude is changed to adapt to various non-standard grooves. The distortion of the groove shape can be solved by adjusting the feeding path of welding torch. The non-uniform change of bottom clearance can be adapted by adjusting swing amplitude slightly in the position of clearance change. The linear change of bottom clearance is mainly solved by adjusting swing amplitude greatly during operation. The effect of swing on welding bead forming is shown in Figure 10.

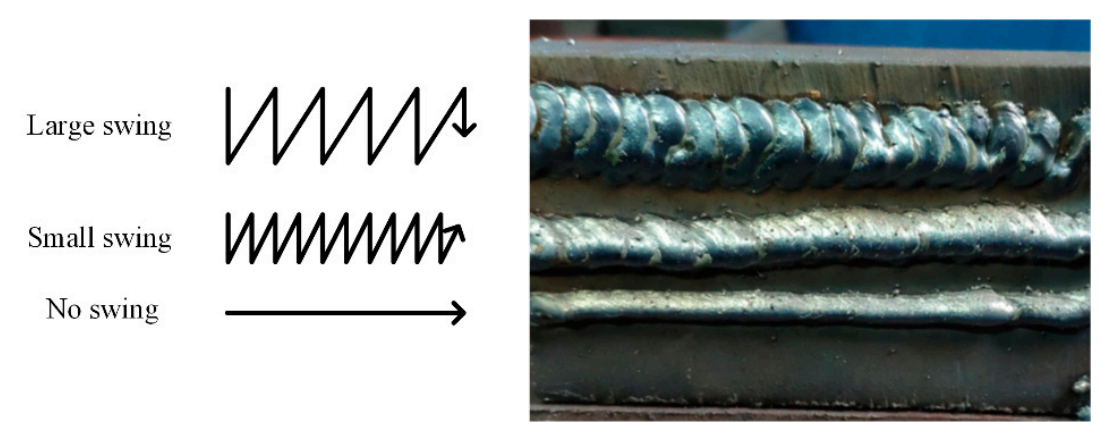

Figure 10. Effect of swing on welding bead formation.

Establish a rectangular coordinate system for the section of steel components. Flame cutting and grinding wheel grinding are commonly used in the groove processing of steel components. Steel plates are fixed in the bottom, in order to ensure easy processing, the gap at the bottom of the groove is smaller than that at the outside. Therefore, we establish a mathematical model for the section of plane quadrilateral, as Figure 11 shows. 


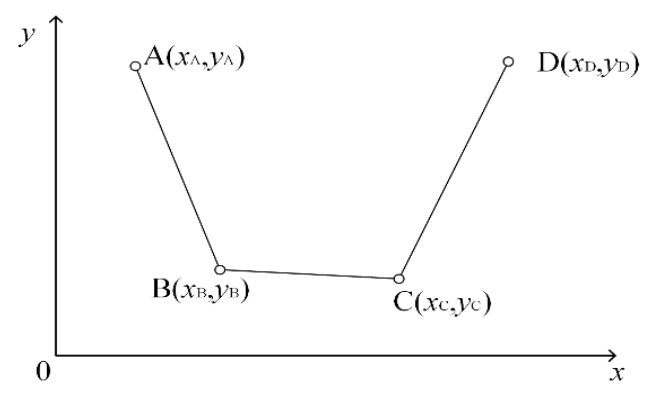

Figure 11. Establishment of coordinate system for single section of groove (welding operations are carried out in turn along the $\mathrm{x}$-axis during cross welding).

\subsection{Extraction of Groove Geometry Information}

Extract geometric information of groove according to coordinates of feature points collected by testing equipment including plate thickness $h$, bottom clearance $a$ and the groove angle of steel components $\alpha$.

The plate thickness $h$ have been manually confirmed before welding operation, or can be obtained by calculating the average thickness of steel frame plates on both sides.

$$
h=\left[\left(y_{A}-y_{B}\right)+\left(y_{D}-y_{C}\right)\right]
$$

Compare it with the parameters of steel components after calculating the thickness of multi-section plate to confirm the data are correct.

Bottom clearance $a$ is the distance between two points at the bottom.

$$
a=\sqrt{\left(x_{B}-x_{C}\right)^{2}+\left(y_{B}-y_{C}\right)^{2}}
$$

Groove angle of steel components $\alpha$ reference object is integral steel member, that is, the angle between the side and the $x$-axis of the coordinate system.

$$
\begin{aligned}
& \alpha_{1}=\arctan \frac{y_{A}-y_{B}}{x_{A}-x_{B}}, \\
& \alpha_{2}=\arctan \frac{y_{D}-y_{C}}{x_{D}-x_{C}},
\end{aligned}
$$

Let the current section be the K section, the current section's plate thickness $h_{k}$, the bottom clearance $a_{k}$ and the groove angle of steel components $\alpha_{1 k}, \alpha_{2 k}$ are acquired. The characteristics of groove parameters are shown in Figure 12.

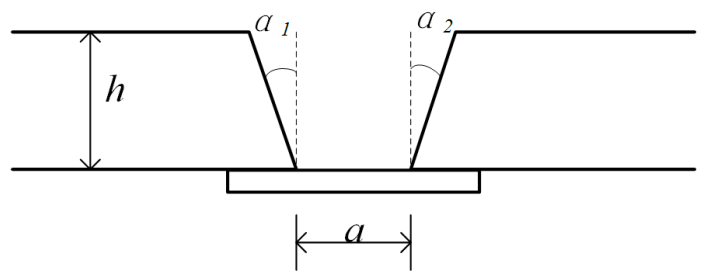

Figure 12. Groove Geometry Information.

The extracted feature parameters can be used not only to match the welding parameters of the expert database, but also to generate the torch path by an intelligent path generation algorithm. 


\subsection{Setting of Welding-Related Parameters}

The width $b$ and height $c$ of each weld are determined according to the welding process parameters and the grinding quantity after the completion of each weld. The welding position and wire size also affect them. All the above parameters should be determined before construction for welding of steel components with specified position, thickness and groove type. The angle of welding torch swing has also been determined. The welding-related parameters are shown in Figure 13.

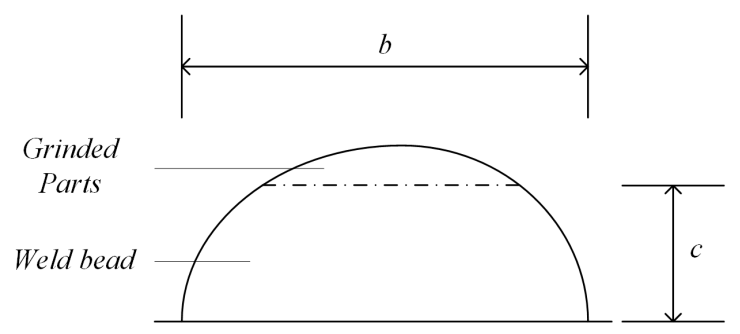

Figure 13. Welding-related parameters.

\subsection{Calculation of Specific Path Coordinates}

(1) Calculation of Layer Number of Weld Bead

The welding form of steel members is multi-layer and multi-pass welding, as shown in Figure 14.

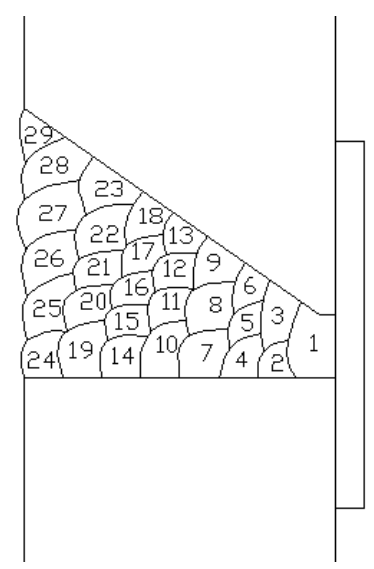

Figure 14. Groove welding layer and track indication.

The number of weld bead layers for each section of the groove of qualified steel components should be uniform and integer. Divide the thickness of the plate by the height of each layer of weld. The number of weld bead layers can be obtained by adaptive treatment.

$$
n=\frac{h}{b}
$$

Rounding $n$, if the remainder is greater than $\frac{b}{2}$, then $n=n+1$.

(2) Calculate the number of paths per layer

The first step is to calculate the position of each layer of bead. Set as number $i$ layer's position. 
The weld bead coordinates of the first layer and the third channel are recorded as $E_{i j}$. The intersection of $\mathrm{AB}$ and $\mathrm{CD}$ edges are as $E_{i 1}$ and $E_{i m_{i}}$. The equation of the straight line where $\mathrm{BC}$, $\mathrm{AB}$ and $\mathrm{CD}$ are located are:

$$
\left\{\begin{array}{l}
\frac{x-x_{B}}{x_{C}-x_{B}}=\frac{y-y_{B}}{y_{C}-y_{B}} \\
\frac{x-x_{A}}{x_{B}-x_{A}}=\frac{y-y_{A}}{y_{B}-y_{A}} \\
\frac{x-x_{C}}{x_{D}-x_{C}}=\frac{y-y_{C}}{y_{D}-y_{C}}
\end{array},\right.
$$

Considering the actual welding operation, steel components should be treated with anti-deformation, so $\mathrm{AB}$ and $\mathrm{CD}$ will not be perpendicular to coordinate system $\mathrm{x}$-axis.

The coordinates of the two end points can be obtained:

$$
\begin{gathered}
E_{i 1}\left(x_{B}+\frac{i-1}{n}\left(x_{A}-x_{B}\right), y_{B}+\frac{i-1}{n}\left(y_{A}-y_{B}\right)\right), \\
E_{i m_{i}}\left(x_{C}+\frac{i-1}{n}\left(x_{D}-x_{C}\right), y_{B}+\frac{i-1}{n}\left(y_{D}-y_{C}\right)\right),
\end{gathered}
$$

Calculate the length of $E_{i 1} E_{i m_{i}}$ :

$$
d_{i}=\left|E_{i 1} E_{i m_{i}}\right|
$$

Number of paths per layer:

$$
m_{i}=\frac{d_{i}}{c}+1,
$$

Rounding $m_{i}$, if the remainder is greater than $\frac{c}{2}$, then $m_{i}=m_{i}+1$.

(3) Calculating the coordinates of each weld path in a single section

$$
E_{i j}\left(x_{B i}+\frac{1}{m_{i}}\left(x_{C i}-x_{B i}\right), y_{B i}+\frac{1}{m_{i}}\left(y_{C i}-y_{B i}\right)\right),
$$

\subsection{Multi-Section Method for Welding Path Planning}

The groove is divided into $l$ sections according to the total length of groove and the position of surface features. The section division of the groove is shown in Figure 15.

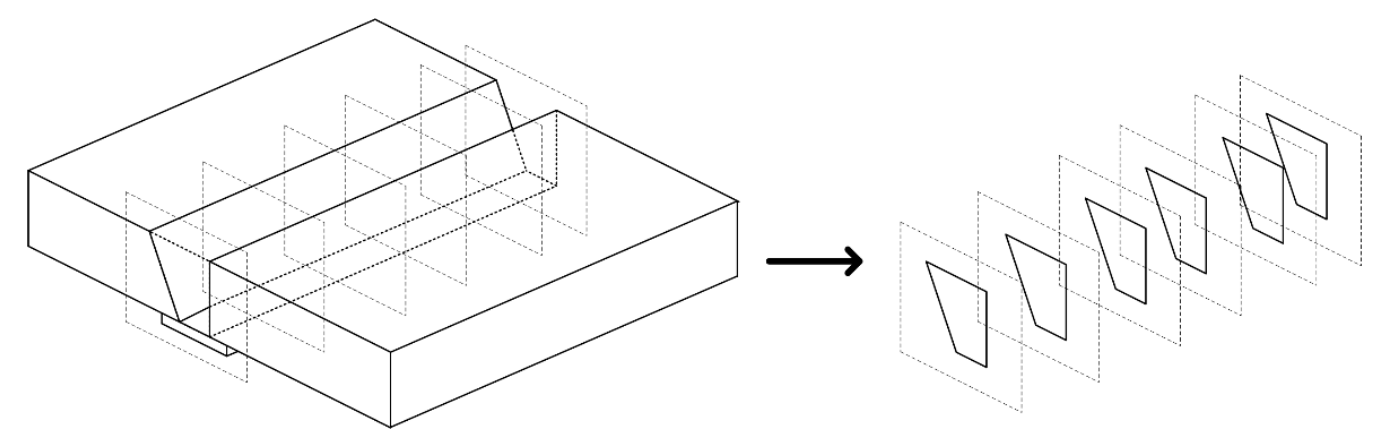

Figure 15. Multi-section division of groove.

When there is Section Counting $k$ satisfied $1 \ll k \ll l$ and $k$ is an integer. The coordinate of weld bead of section $k$, layer $i$, track $j$ is obtained:

$$
\begin{gathered}
E_{i j k}\left(x_{B k}+\frac{i-1}{n}\left(x_{A k}-x_{B k}\right)+\frac{j-1}{m_{i}}\left\{\left(x_{C k}-x_{B k}\right)+\frac{i-1}{n}\left[\left(x_{D k}-x_{C k}\right)-\left(x_{A k}-x_{B k}\right)\right]\right\},\right. \\
\left.y_{B k}+\frac{i-1}{n}\left(y_{A k}-y_{B k}\right)+\frac{j-1}{m_{i}}\left\{\left(y_{C k}-y_{B k}\right)+\frac{i-1}{n}\left[\left(y_{D k}-y_{C k}\right)-\left(y_{A k}-y_{B k}\right)\right]\right\}\right),
\end{gathered}
$$


As shown in Figure 16, set the $i j k$-nested loops in programs and set the welding torch preparation posture before each weld bead starring and welding torch lift-off posture after finishing. Set separate torch path and inverse solution algorithms for feature parts. The whole provision can automatically complete all the work from detecting weld bead layout to path planning and finally complete welding operation.



Figure 16. Path generation of welding torch with multi-section interpolation.

\subsection{Approach of Non-Standard Groove Welding}

In case of groove shape distortion, the path generated by the algorithm has the same effect as the manual operation.

In case of a non-uniform variation of bottom clearance, the path generated by the algorithm will adjust the position where the gap changes to ensure that the melted wire can fill the current layer of the groove.

In the case of a unidirectional linear variation of bottom clearance, different from the method of greatly adjusting swing amplitude in the whole welding process, the method used in this algorithm is to add new paths to melt wire and fill grooves without changing the swing range of single pass weld.

\section{Experiments and Results}

\subsection{Experimental Scheme}

The experimental object is carbon steel component specimens, the test plate and the steel liner are joined by spot welding. The groove form is L groove, and the welding position is transverse welding. The form of non-standard groove is linear change of bottom clearance in one direction.

After specimen preparation and camera calibration, the groove is detected by the visual inspection detection bench, and the multi-section characteristic parameters of the groove are obtained by processing the collected data. Using the designed algorithm, the extracted multi-section characteristic parameters are processed and the interpolation coordinate points of the torch path are obtained. The coordinates are imported into software to get the simulation path. Finally, the test plate is welded according to the path generated by the algorithm. After welding, the feasibility and correctness of the algorithm are verified by the quality inspection of the specimens. 


\subsection{Detection Bench and Images Processes}

The detection bench is mainly composed of visual system and auxiliary equipment. The vision system includes CCD and linear structured light. The supporting device includes a support for fixing and placing the vision system and an auxiliary shifter for placing the tested object. The composition of the detection bench is shown in Figure 17.

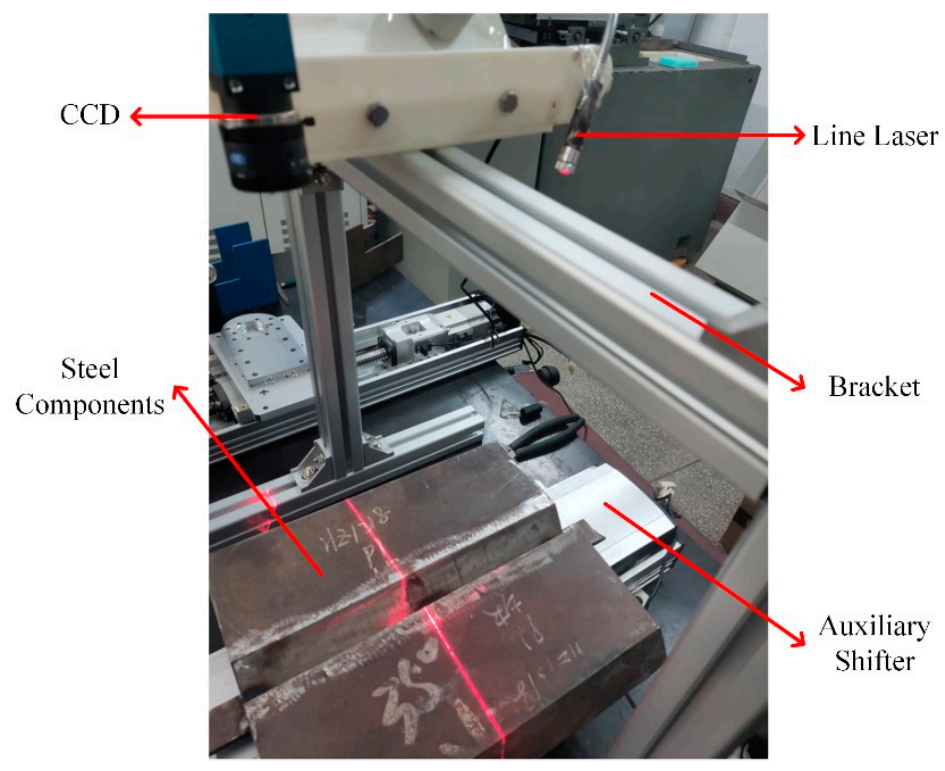

Figure 17. Structure of the detection stand.

The whole system can be applied to automatic welding equipment. In order to simulate the movement of steel component fixed detection system in actual operation, the vision system is fixed and the tested piece is placed on the auxiliary shifter. The detection time line laser irradiates the surface of the specimen under test, the shifter drives the specimen to move, and the camera collects pictures with a certain rate of evaluation. The complex image is processed to extract multi-section feature parameters.

The feature points of the current section groove can be obtained by clipping, contrast enhancement, noise removal and extracting the center line of the image, and the data of reconstructing the whole groove can be obtained by multi-group image processing. The specific image processing steps are shown in Figure 18.

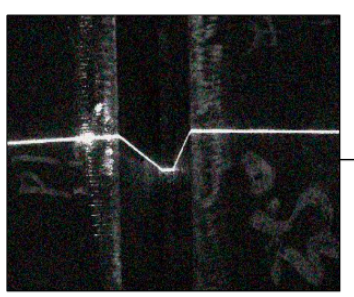

Clipped image

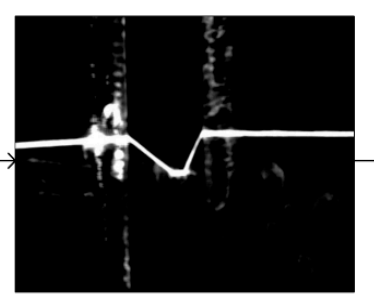

Contrast

enhancement

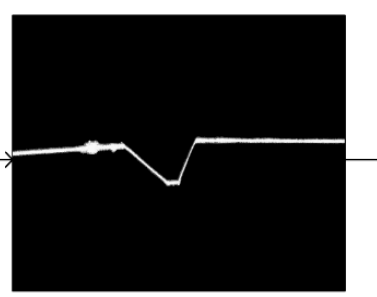

Noise

Removal

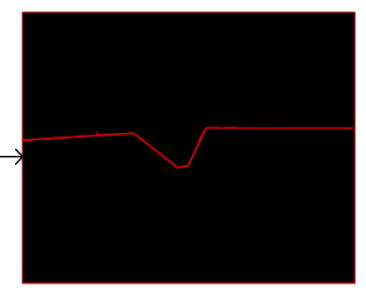

Extraction of

Bar Center

Figure 18. Image processing. 


\subsection{Simulation Results}

According to the path survival algorithm, the software is compiled. The multi-section characteristic parameters of groove are imported into the software to obtain the torch path coordinates. Then the coordinates are connected to the simulation interpolation sequentially, and the torch path simulation diagram is obtained. The simulation results of 1 st, 2 nd layer and the whole groove are shown in Figures 19-21.

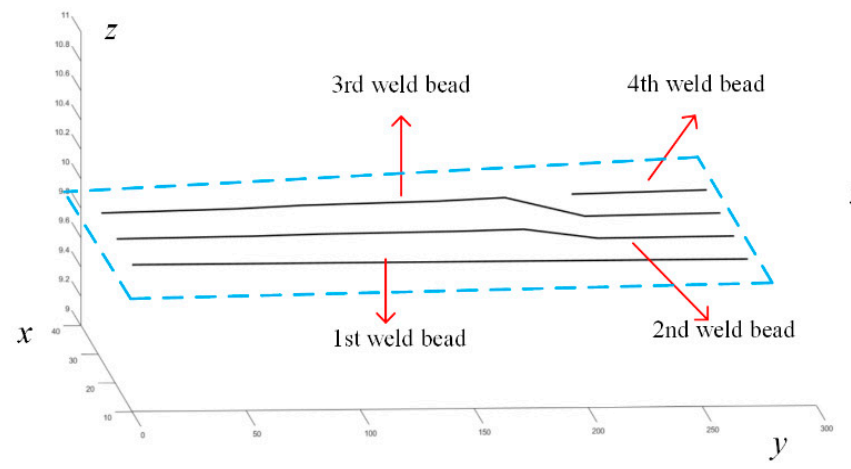

(a)

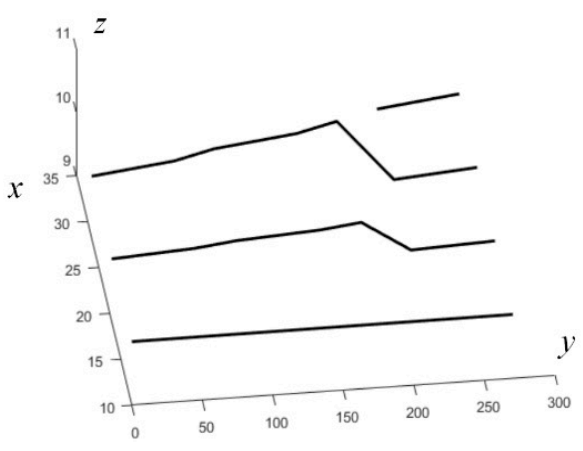

(b)

Figure 19. (a) Simulation results of the first layer welding path (the blue part is the groove outline); (b) Simulation results in a different angle.



(a)

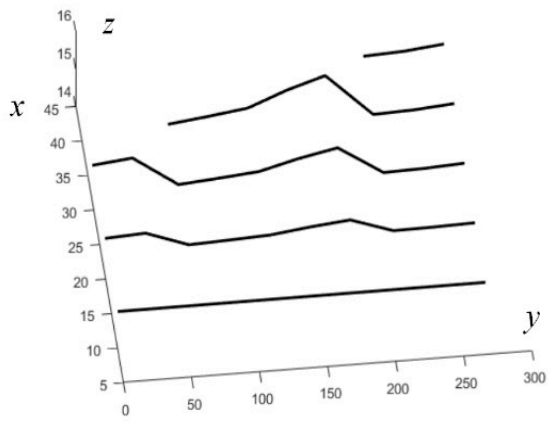

(b)

Figure 20. (a) Simulation results of the first two layers welding path (the blue part is the groove outline); (b) Simulation results of the path generation of separate second layer.

From the simulation results, it can be seen that the road strength generated by the algorithm has the following characteristics:

(1) Make sure that each layer of bead and edge are closely occluded;

(2) The bead can be adaptively filled into the groove with linear change of gap in one direction without overlapping in space;

(3) The distance between beads should be controlled in a reasonable range, so that the distance between beads would not be too close. 


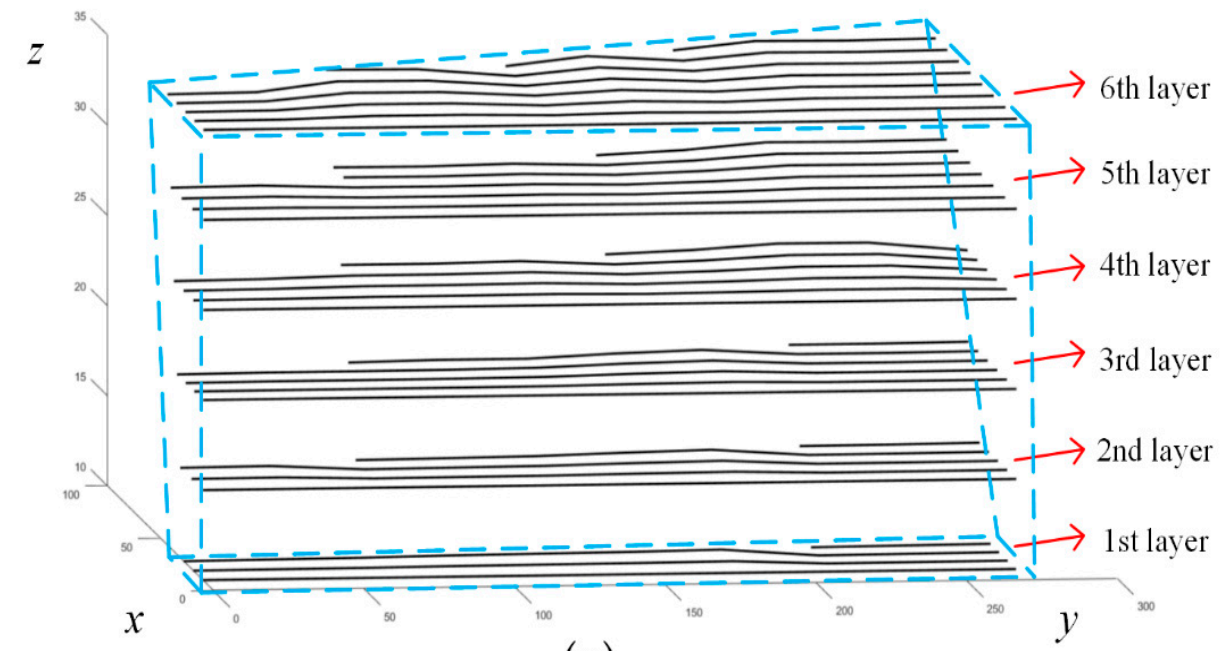

(a)

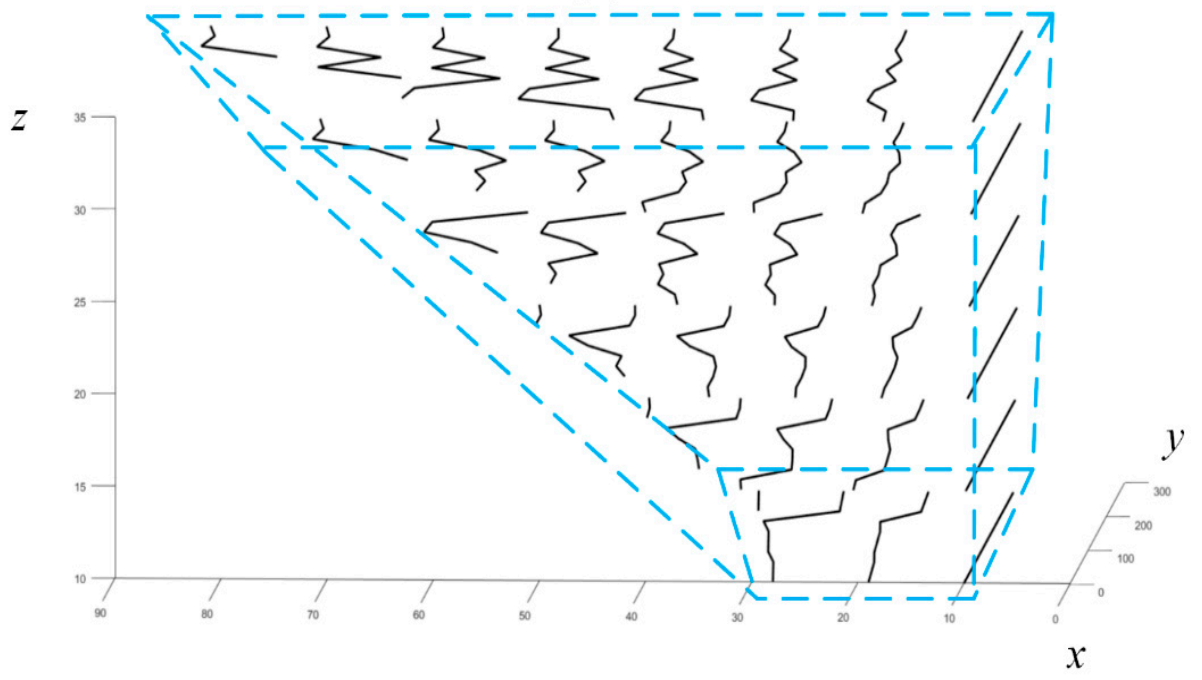

(b)

Figure 21. (a) Simulation results of path generation (the blue part is the groove outline); (b) Simulation results of path generation in section direction.

\subsection{Experimental Process}

Welding experiments were carried out according to the simulated trajectories. In order to distinguish it from the traditional welding process, there is almost no swing motion perpendicular to the feeding direction of the torch during the experiment. After each layer of welding is completed, the edge is observed by naked eye. The spatter of weld slag which adheres to the edge of steel components affects the cleaning by manual operation, and the cleaning of welding torch nozzle is also manual operation. The material and welding parameters of the test plate used in the experiment are shown in Tables 2 and 3.

Table 2. Chemical constitution and mechanical properties of test plate.

\begin{tabular}{cccccccccc}
\hline \multirow{2}{*}{ Parameter Indicators } & $\mathbf{C}$ & $\mathbf{M n}$ & $\mathbf{S i}$ & $\mathbf{S}$ & $\mathbf{P}$ & $\boldsymbol{\sigma s}$ & $\boldsymbol{\sigma b}$ & $\Delta \mathbf{s}$ & $\mathbf{A k v}$ \\
\cline { 2 - 10 } & $\leq \%$ & $\leq \%$ & $\leq \%$ & $\leq \%$ & $\leq \%$ & $\mathbf{( M P a})$ & $\mathbf{( M P a )}$ & $\%$ & $\mathbf{J}$ \\
\hline Standard & 0.2 & 1.6 & 0.55 & 0.015 & 0.035 & $\geq 350$ & $490-650$ & $\geq 20$ & $\geq 34$ \\
Actual & 0.16 & 1.51 & 0.29 & 0.0025 & 0.018 & 437 & 574 & 27 & 205 \\
\hline
\end{tabular}


Table 3. Welding parameters.

\begin{tabular}{cccccc}
\hline Weld Type & $\begin{array}{c}\text { Welding Wire } \\
(\mathbf{m m})\end{array}$ & $\begin{array}{c}\text { Shielding Gas } \\
(\mathbf{L} / \mathbf{m i n})\end{array}$ & $\begin{array}{c}\text { Electric } \\
\text { Current }(\mathbf{A})\end{array}$ & $\begin{array}{c}\text { Voltage } \\
\mathbf{( V )}\end{array}$ & $\begin{array}{c}\text { Welding Speed } \\
\text { (cm/min) }\end{array}$ \\
\hline Root weld & 1.2 & 35 & 230 & 28 & 35 \\
Filler passes & 1.2 & 40 & 260 & 33 & 35 \\
\hline
\end{tabular}

\subsection{Welding Results}

\subsubsection{Welding Bead Distribution}

Figure 22(a4,b5) show that the length of some weld bead does not reach the total length of groove. The integration of steel components and specimens can be ensured by adjusting the angle of welding torch when welding the weld that contacts the groove edge.

\subsubsection{Welding Bead Lap Joints}

As shown in Figure 23, for the third layer with obvious features, the welding path generated by the multi-section interpolation path generation algorithm has good filling effect, and the biting of bead and edge also meets the requirements of the specification.

a1

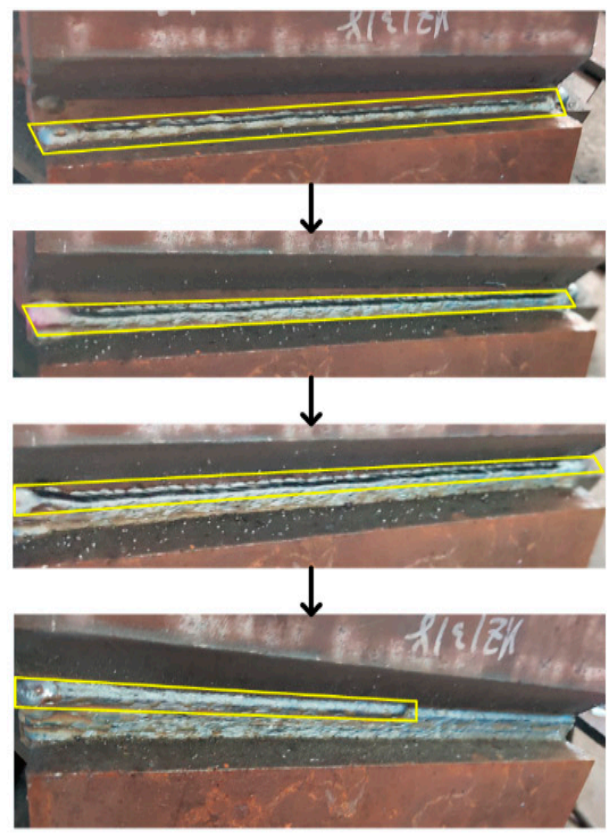

(a)

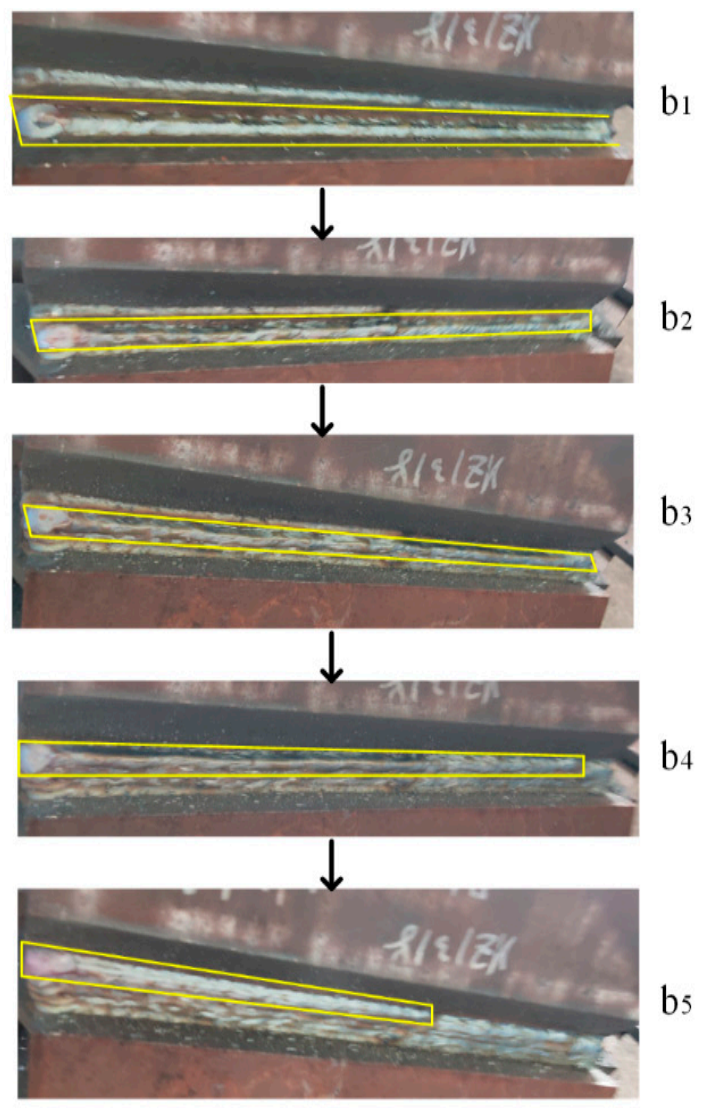

(b)

Figure 22. (a) The first layer welding operation (the yellow-framed part is the current weld bead); (b) The second layer welding operation. 


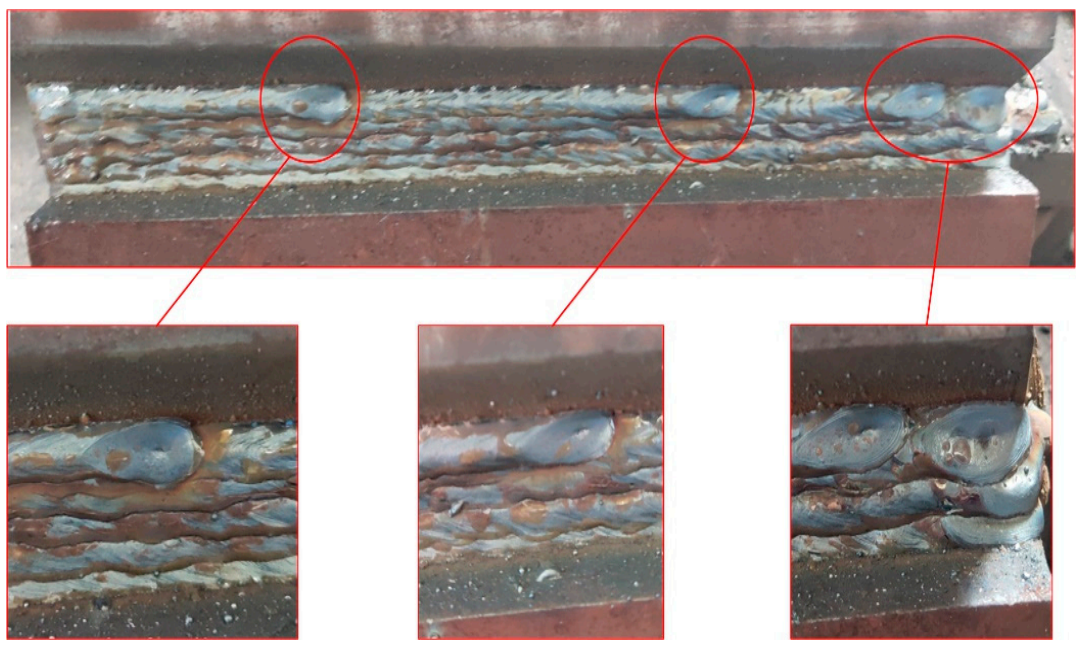

Figure 23. Local close-up of arc closing position of segmental lapping.

\subsubsection{Welding Bead Quality}

Tensile, bending and impact tests are carried out on the welded steel members. The results are shown in Table 4.

Table 4. Welding experimental result.

\begin{tabular}{cccccc}
\hline Specimen Number & $\begin{array}{c}\sigma_{\mathbf{b}} \\
(\mathbf{M P a})\end{array}$ & Bend Diameter & Bend Angle & $\begin{array}{c}\text { Notch } \\
\text { Location }\end{array}$ & $\begin{array}{c}\text { Akv } \\
\text { J }\end{array}$ \\
\hline 001 & 521 & 30 & 180 & Weld bead & 137 \\
\hline
\end{tabular}

\section{Conclusions}

A vision-based multi-section interpolation algorithm for non-standard groove welding path generation is discussed. Firstly, the non-standard grooves are classified and the causes are discussed. Then, the system scheme of intelligent automatic welding equipment is analyzed, a visual inspection testbed is built, and the coordinates of feature points are obtained by processing the collected groove images. The multi-section interpolation algorithm of non-standard groove welding path generation is used to generate the welding torch path using software simulates the torch path generated by the algorithm. Finally, through the complete experiment of detecting welding, the validity of visual-based multi-section interpolation for the non-standard groove welding path generation algorithm is verified. The major findings are summarized as follows:

(1) An intelligent automatic welding equipment system with functions from detecting welding track generation to completing welding operation was built;

(2) Choose the appropriate visual inspection scheme, inspect the groove, process the image, and obtain the coordinates of feature points of non-standard groove with multi-section;

(3) The coordinates of non-standard groove multi-section feature points obtained by the detection system are imported into the algorithm of path generation, and the path of welding torch is obtained;

(4) The results of welding experiment are in good agreement with the planned results;

(5) The filling of weld seam and edge occlusion effect are good. Welding according to the path generated by the algorithm can meet the quality requirements.

Author Contributions: Conceptualization, H.L. and M.M.; Methodology, S.L. (Shu Liu) and E.A.; Writing-Original Draft Preparation, M.M.; Writing-Review and Editing, Y.Z. and W.Z.; Validation and Data Curation, S.L. (Shuo Li). All authors have read and agreed to the published version of the manuscript. 
Funding: This work was funded by The National Natural Science Foundation of China (Grant no. 51675393), the National Key R\&D Plan, key projects of green building and building industrialization (No. 2017YFC0703903).

Conflicts of Interest: The authors declare no conflict of interest.

\section{References}

1. Keane, M.J.; Siert, A.; Chen, B.T.; Stone, S.G. Profiling mild steel welding processes to reduce fume emissions and costs in the workplace. Ann. Occup. Hyg. 2014, 58, 403-412. [PubMed]

2. Keane, M.J.; Siert, A.; Stone, S.; Chen, B.; Slaven, J.; Cumpston, A.; Antonini, J. Selecting processes to minimize hexavalent chromium from stainless steel welding. Weld. J. 2012, 19, 241-246.

3. Wang, J.; Kalivoda, M.; Guan, J.; Theodore, A.; Sharby, J.; Wu, C.-Y.; Paulson, K.; Es-Said, O. Double shroud delivery of silica precursor for reducing hexavalent chromium in welding fume. J. Occup. Environ. Hyg. 2012, 9, 733-742. [CrossRef] [PubMed]

4. Norberto, P.J.; Bölmsjo, G.; Loureiro, A. Welding robots: Technology, system issues and applications. Ind. Robot 2005, 10, 45-55.

5. Micallef, K.; Fang, G.; Dinham, M. Automatic seam detection and path planning in robotic welding. In Lecture Notes in Electrical Engineering; Springer: Berlin/Heidelberg, Germany, 2011; pp. 23-32.

6. Xu, Y.; Yu, H.; Zhong, J.; Lin, T.; Chen, S. Real-time seam tracking control technology during welding robot GTAW process based on passive vision sensor. J. Mater. Process. Technol. 2012, 212, 1654-1662. [CrossRef]

7. Bračun, D.; Sluga, A. Stereo vision based measuring system for online welding path inspection. J. Mater. Process. Technol. 2015, 223, 328-336. [CrossRef]

8. Boillot, J.P.; Noruk, J. The benefits of laser vision in robotic arc welding-Laser vision weld inspection can help reduce destructive testing costs. Weld. J. 2002, 32-34.

9. Hiramoto, S.; Moriyasu, M.; Ohmine, M. Development of a multipass welding program for arc welding robots and its application to heavy electrical components. Weld. Int. 1992, 6, 26-31. [CrossRef]

10. Tang, X.-H.; Drews, P. 3D-visualized offline-programming and simulation system for industrial robots. Trans. China Weld. Inst. 2005, 2.

11. Chen, S.B.; Zhang, Y.; Qiu, T.; Lin, T. Robotic welding systems with vision-sensing and self-learning neuron control of arc welding dynamic process. J. Intell. Robot. Syst. 2003, 36, 191-208. [CrossRef]

12. Yang, C.; Ye, Z.; Chen, Y.; Zhong, J.; Chen, S. Multi-pass path planning for thick plate by DSAW based on vision sensor. Sens. Rev. 2014, 34, 416-423. [CrossRef]

13. Ye, Z.; Fang, G.; Chen, S.; Zou, J.J. Passive vision based seam tracking system for pulse-MAG welding. Int. J. Adv. Manuf. Technol. 2013, 67, 1987-1996. [CrossRef]

14. Fan, C.; Lv, F.; Chen, S. Visual sensing and penetration control in aluminum alloy pulsed GTA welding. Int. J. Adv. Manuf. Technol. 2009, 42, 126-137. [CrossRef]

15. Kovnatsky, A. Feature points in image, keypoint extraction. Org. Biomol. Chem. 2009, 7, 2077-2081.

16. Li, B.; Mu, C.; Wang, T.; Peng, Q. Revised version of a JCIT paper-comparison of feature point extraction algorithms for vision based autonomous aerial refueling. arXiv 2014, arXiv:1405.6163.

17. Yuba, Y.; Hirai, H.; Tsutsumi, K.; Watari, K. Rotated checker-pattern projection and its cross-point extraction and tracking for 3-D object profilometry. Syst. Comput. Jpn. 2010, 20, 53-61. [CrossRef]

18. Sorokin, D.V.; Mizotin, M.M.; Krylov, A.S. Gauss-laguerre keypoints extraction using fast hermite projection method. In International Conference Image Analysis and Recognition; Springer: Berlin/Heidelberg, Germany, 2011; pp. 284-293.

19. Hu, X.; Zhang, Z.; Li, J. Linear feature extraction using adaptive least-squares template matching and a scalable slope edge model. Int. J. Remote Sens. 2009, 30, 3393-3407. [CrossRef]

20. Hochstenbach, M.E. Harmonic and refined extraction methods for the singular value problem, with applications in least squares problems. Bit Numer. Math. 2004, 44, 721-754. [CrossRef]

(C) 2020 by the authors. Licensee MDPI, Basel, Switzerland. This article is an open access article distributed under the terms and conditions of the Creative Commons Attribution (CC BY) license (http://creativecommons.org/licenses/by/4.0/). 